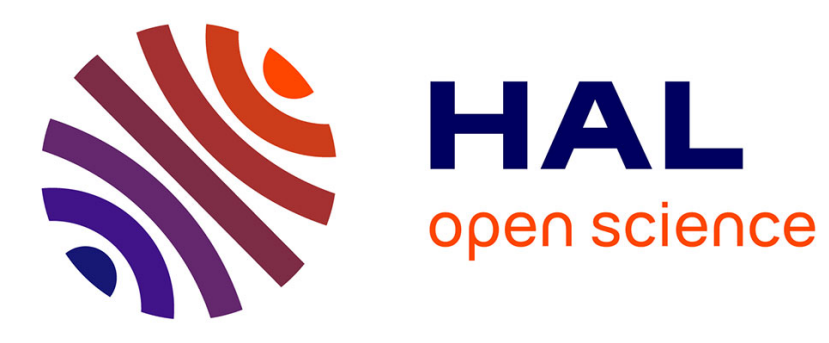

\title{
Spatiotemporal Analysis of Brain Functional Connectivity
}

\author{
Ahmad Mheich, Mahmoud Hassan, Olivier Dufor, Mohamad Khalil, Claude \\ Berrou, Fabrice Wendling
}

\section{- To cite this version:}

Ahmad Mheich, Mahmoud Hassan, Olivier Dufor, Mohamad Khalil, Claude Berrou, et al.. Spatiotemporal Analysis of Brain Functional Connectivity. MBEC 2014: 6th European Conference of the International Federation for Medical and Biological Engineering, Sep 2014, Dubrovnik, Croatia. pp.934-937, 10.1007/978-3-319-11128-5_232 . inserm-01071395

\section{HAL Id: inserm-01071395 https://www.hal.inserm.fr/inserm-01071395}

Submitted on 4 Oct 2014

HAL is a multi-disciplinary open access archive for the deposit and dissemination of scientific research documents, whether they are published or not. The documents may come from teaching and research institutions in France or abroad, or from public or private research centers.
L'archive ouverte pluridisciplinaire HAL, est destinée au dépôt et à la diffusion de documents scientifiques de niveau recherche, publiés ou non, émanant des établissements d'enseignement et de recherche français ou étrangers, des laboratoires publics ou privés. 


\title{
Spatiotemporal analysis of brain functional connectivity
}

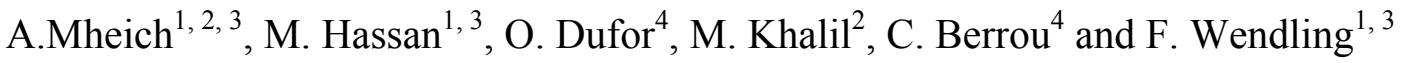 \\ ${ }^{1}$ INSERM, U1099, Rennes, F-35000, France \\ ${ }^{2}$ AZM center-EDST, Lebanese University, Tripoli, Lebanon \\ ${ }^{3}$ Université de Rennes 1, LTSI, F-35000, France \\ ${ }^{4}$ Télécom Bretagne (Institut Mines-Télécom), UMR CNRS Lab-STICC, Brest, France
}

\begin{abstract}
Brain functions are based on interactions between neural assemblies distributed within and across distinct cerebral regions. During cognitive tasks, these interactions are dynamic and take place at the millisecond time scale. In this context, the excellent temporal resolution $(<1 \mathrm{~ms})$ of the Electroencephalographic -EEG- signals allows for detection of very short-duration events and therefore, offers the unique opportunity to follow, over time, the dynamic properties of cognitive processes.

In this paper we propose a new algorithm to track the functional brain connectivity dynamics. During picture recognition and naming task, this algorithm aims at segmenting high resolution (hr) EEG functional connectivity microstates. The proposed algorithm is based on the K-means clustering of the connectivity graphs obtained from the Phase Locking Values (PLV). Results show that the algorithm is able to track the brain functional connectivity dynamics during picture naming task.
\end{abstract}

Keywords-High resolution EEG, PLV, K-means, functional connectivity dynamics.

\section{INTRODUCTION}

A key challenge in neuroscience is to identify distinctive networks underlying specific brain functions, from data provided by neuroimaging techniques, which can be either structural (diffusion imaging, DTI) or functional (electroencephalography, -EEG magnetoencephalography -MEG- or even functional magnetic resonance imaging fMRI-). These neuroimaging techniques can be used to identify brain networks involved in normal brain functions(behavioral response to stimulus, learning, memory) as well as in neurological disorders like epilepsy, Alzheimer, Schizophrenia[1].

In this context, fMRI has significantly evolved over the past two decades and is now commonly used to characterize brain connectivity. However, the short duration of cognitive processes ( $\sim 500 \mathrm{~ms}$ for the picture naming, for example) requires the use of techniques that have a very high time resolution (on the order of $\mathrm{ms}$ ), which is not the case of fMRI $(\sim 1 s)$, although it has the advantage of having excellent spatial resolution.
Several studies have indicated that the use of electroencephalography (EEG) -which provides scalp signals at excellent temporal resolution (sub-millisecond) - combined with appropriate signal processing techniques can bring relevant information about normal networks activated during cognitive activity[2] or about disturbed networks associated for instance with tumors[3].

This excellent temporal resolution allows us to analyze the dynamic properties of the cognitive process, a challenging issue so far addressed in a few studies only. $\operatorname{In}[4,5]$, proposed algorithms were based on the Event Related Potentials (ERPs) amplitude. However, these algorithms do not take into account the connectivity between signals (electrode space) or brain regions (source space). Other methods rely on the decomposition of EEG measurements into topographic and time-frequency elements. These methods based on frequency analysis, show that a large set of data can be represented by a small number of topographic distributions [6].Again, these methods ignore the connectivity aspects.

Regarding the results based on the connectivity analysis, most of reported methods make use of a constant time window to track the EEG dynamic connectivity. This window is chosen empirically or based on a priori information about the analyzed task [2]. A few attempts have been recently reported to avoid this constraint $[7,8]$. However, most of the proposed algorithms are not adequate to track changes over very short durations (as in the case of responses evoked by visual stimuli).

In this paper, we propose a new algorithm to track the brain functional connectivity dynamics during picture naming task. The proposed algorithm is based on the K-means clustering of the connectivity graphs obtained by the trial by trial Phase Locking Values (PLV) method.

\section{Materials AND MEtTHOds}

\section{A. DATA}

Six subjects were shown pictures on a screen using EPrime 2.0 software (Psychology Software Tools, Pittsburgh, PA). They were asked to name the displayed objects. The 
148 images were selected from a database of 400 pictures standardized for French[9] and were used during two sessions (about eight minutes each) of 74 stimuli. The brain activity was recorded using an hr-EEG system (256 electrodes, EGI, Electrical Geodesic Inc.). EEG signals were collected at a $1 \mathrm{kHz}$ sampling frequency and were bandpass filtered between 3 and $45 \mathrm{~Hz}$. Each trial was visually inspected, and epochs contaminated by eye blinking, movements or any other noise source were rejected and excluded from the analysis performed using the EEGLAB open source toolbox[10]. This study was approved by the National Ethics Committee for the Protection of Persons (CPP), (conneXion study, agreement number 2012-A0122736, promoter: Rennes University Hospital). We excluded the electrodes located on the face as well as the few electrodes showing too high impedance. Overall, in our hr-EEG montage, 180 (over 256) electrodes were retained as providing excellent quality signals over all subjects.

\section{B. Functional connectivity measure}

Functional connectivity is defined as the temporal correlation (wide sense) among the activity of different neuronal assemblies. Several methods have been proposed to characterize the brain functional connectivity. In this paper we used the phase locking value (PLV) method proposed by Lachaux et al. [11] to measure synchronization on a trial by trial basis. For each channel pair, $x$ and $y$, at time $t$, and for all the trials $(n=1 \ldots N), \mathbf{P L V}$ is defined as:

$$
\mathrm{PLV}_{x y}=\frac{1}{N}\left|\sum_{n=1}^{N} \varphi_{x}-\varphi_{y}\right|
$$

Where $\varphi_{x}$ and $\varphi_{y}$ are the unwrapped phases of signals $x$ and $y$ respectively. We applied a normalization procedure so that the $\mathrm{PLV}_{x y}$ values were compared with the $200 \mathrm{~ms}$ baseline preceding the presentation of the image. Let $\mu_{x y}$ and $\sigma_{x y}$ be the mean and standard deviation computed from a 200ms pre-stimulus baseline. The normalized PLV is then computed as $\operatorname{PLV}_{x y}=\left(\operatorname{PL} \chi_{x y}-\right)_{x y} \sigma_{x y}$. The connectivity matrices are computed in the low gamma frequency band $(30 \mathrm{~Hz}-45$ $\mathrm{Hz}$ ). A thresholding procedure is then applied on the functional connectivity values in order to retain a small fraction $(10 \%)$ of the strongest functional connections, as discussed in [12].

\section{Segmentation algorithm}

The proposed algorithm can be summarized as follows:
1- Compute the adjacency matrices for each subject using PLV to obtain $T$ connectivity graphs. The instantaneous averaged connectivity PLV (t) is computed over all the subjects.

2- $\quad$ Select randomly $K$ graphs ( $K$ varies from 3 to-10) from the $T$ graphs. To avoid the choice of very close graphs, an additional constraint has been added which consists in rejecting the $K$ graphs if the time interval between two graphs in any pairs is less than $30 \mathrm{~ms}$.

3- Calculate the spatial correlation (sC) between each of the $K$ and $T$ graphs. $\mathbf{s C}$ is computed based on PLV(t). For two pairwise PLV P and Q, at instant $t$, $\mathbf{s C}(\mathbf{t})$ is defined as:

$$
\mathrm{sC}_{P, Q}(t)=\frac{\sum_{i=1}^{M} P_{i} \cdot Q_{i}}{\sqrt{\sum_{i=1}^{M} P_{i}^{2}} \cdot \sqrt{\sum_{i=1}^{M} Q_{i}^{2}}}
$$

Where $M$ is the number of combinations $(180 \times(180-$ 1)/2).

4- From these spatial correlation values, the Global Explained Variance (GEV) is calculated as defined in[5]. While GEV is unstable (i.e. doesn't reach its highest value over the iterations), the template maps are redefined averaging all the graphs yielding to the same cluster. When reaching the highest GEV, $K+1$ template maps are then selected and all the above procedure is repeated. To choose the optimal number of template graphs, we use a method based on the Cross Validation (CV) criterion as introduced in[5] which is the ratio between GEV and the degrees of freedom for a given set of graphs. Its minimum gives the optimal number of segments. Finally, an additional constraint is used to prevent the small clusters, a given connectivity graph must "survive" for at least $30 \mathrm{~ms}$.

5- The result obtained in the averaged data is then compared with the moment-by-moment connectivity graphs of individual subjects PLVs. Each time point is labelled according to the graph with which it best correlates, yielding a measure of graph presence. This procedure referred to as 'fitting' allows for establishing how well a cluster map explains individual activity and its duration.

\section{RESULTS}

To start with, after the random selection of the template graphs, we compute the spatial correlation between these 
template maps and all the graphs. An example of the $\mathbf{s C}$ curves (at each instant) is shown in Fig. 1 for 3 random different instants. The figure shows how $\mathbf{s C}$ is able to detect the spatial correlation between graphs. We can notice also that around the highest correlation peak, the correlation values are higher than temporary distant graphs.

This step is followed by the computation of GEV for different template graphs. In our study the number of clusters varies from 3 to 10 clusters. The lowest $\mathbf{C V}$ value is obtained for a number of clusters equal to 6 .

We then choose this number of clusters to identify the spatiotemporal behavior of the connectivity graphs obtained with the algorithm.

Results are exemplified in Fig. 2. The figure shows the segmentation of ERP signals (180 channels) into 6 clusters. The first cluster corresponds to the period from 0 (onset) to $116 \mathrm{~ms}$. In this cluster, we can observe a network located in the occipital lobe.

A shorter graph is then observed between 117 and $153 \mathrm{~ms}$ with also strong connections at the occipital and temporal lobe. The $3^{\text {rd }}$ cluster is located at $154-190 \mathrm{~ms}$ where strong connections appear in the temporal and parietal lobes. A network appears in the cluster 191-316ms with the presence of connectivity in frontal regions, followed by a cluster (317-480 ms) with dense connectivity in frontal and occipital regions.

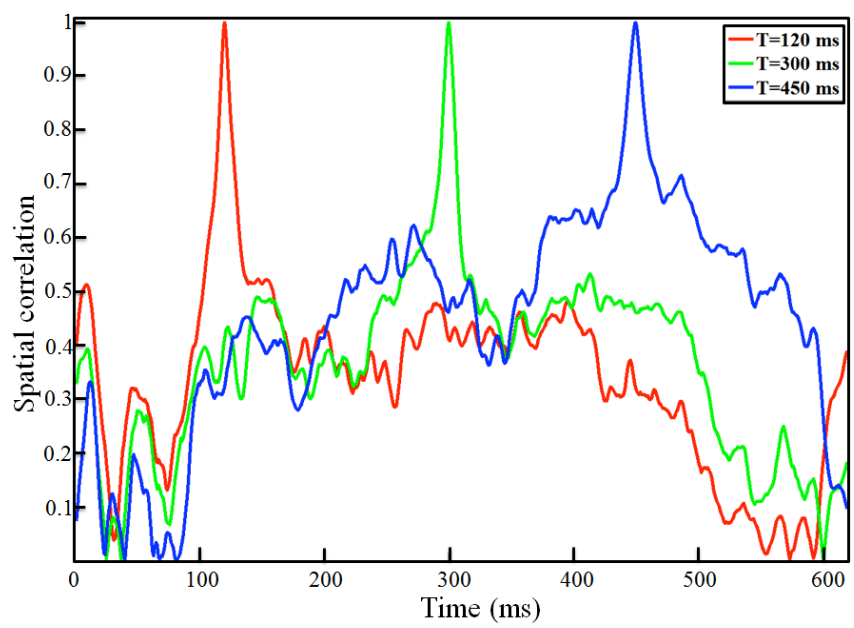

Fig1.Spatial correlation ( $\mathbf{s C})$ of 3 templates $(\mathrm{t}=120, \mathrm{t}=300, \mathrm{t}=450)$ with all the others graphs $(T)$.

The networks (481-620 ms) then become denser in temporal left and frontal regions.

Table 1 shows the results obtained after the 'fitting' process which consists in testing the inter-subject variability. The map presence values are presented in column, the templates (T3, T6) have high values of maps presence between subjects $(73 \%, 78 \%)$ and low values in the template $(\mathrm{T} 1, \mathrm{~T} 4)$.

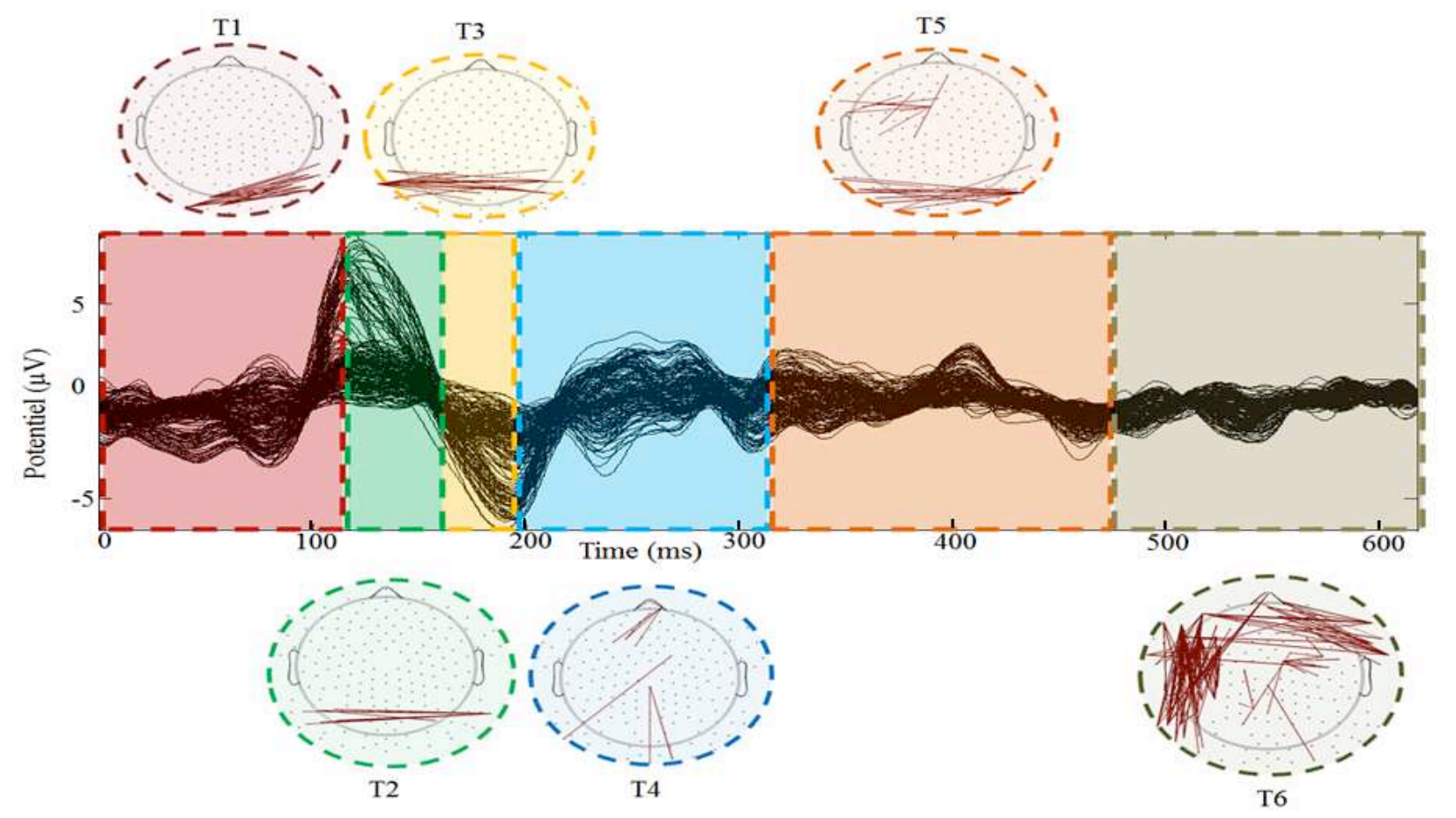

Fig 2.Event Related Potentials (180 electrodes) for the picture naming task and temporal distribution of the functional connectivity graphs revealed by spatio-temporal segmentation algorithm detailed in the paper. Connectivity graphs and their specific time windows are showed by each rectangle. 
Table1

Mean and standard deviation of the map presence after the fitting step.

\begin{tabular}{|c|c|c|c|c|c|c|}
\hline & $\mathrm{T} 1$ & $\mathrm{~T} 2$ & $\mathrm{~T} 3$ & $\mathrm{~T} 4$ & $\mathrm{~T} 5$ & $\mathrm{~T} 6$ \\
\hline $\begin{array}{c}\text { Map } \\
\text { presence }\end{array}$ & $31 \% \pm 16$ & $41 \% \pm 38$ & $73 \% \pm 30$ & $23 \% \pm 28$ & $39 \% \pm 22$ & $78 \% \pm 32$ \\
\hline
\end{tabular}

\section{Discussion}

Preliminary results were presented to demonstrate that the proposed algorithm can be used to track the dynamics of the brain functional connectivity. Very interestingly, results are qualitatively consistent with the state of the art of the analyzed task regarding the activation starting in the occipital lobes during picture recognition followed by activation in the temporo-frontal regions[13].One of the challenge faced by our study is to specify the maximal number of clusters. The increase of the maximal number of clusters increases the computation cost. In our study we used 10 clusters. A classical and still unsolved difficult question relates to the setting of threshold values applied on the connectivity matrices. In our study, the chosen threshold value was $10 \%,[12]$. Other approaches can be explored like those based on surrogate data[2], although requiring an even higher computation time. Finally, this algorithm is a good start to then compare the dynamics of the brain connectivity for two different cognitive tasks such as the difference between spelling[14] and naming task which is our ongoing work. Also the algorithm will be applied on EEG source connectivity graphs to track brain functional connectivity dynamics at cortical source level.

\section{v. Conclusion}

In this paper we proposed a new algorithm to track the functional brain connectivity dynamics and segment hrEEG signals recorded during picture recognition and naming task using $K$-means clustering of the connectivity graphs. This algorithm shows good performance to demonstrate the stability of the functional brain connectivity overs some short periods of time and to segment the cognitive process into functional connectivity microstates.

\section{ACKNOWLEDGEMENTS}

This work has received support from European Research Council (ERC) and from French government granted to the CominLabs excellence laboratory and managed by the $\mathrm{Na}$ tional Research Agency in the "Investing for the Future" program under reference Nb. ANR-10-LABX-07-01.

\section{REFERENCES}

1. Stam, C. J., de Haan, W., Daffertshofer, A., Jones, B. F., Manshanden, I., van Cappellen van Walsum, A. M., Montez, T., Verbunt, J. P., de Munck, J. C., van Dijk, B. W., Berendse, H. W., and Scheltens, P., "Graph theoretical analysis of magnetoencephalographic functional connectivity in Alzheimer's disease," Brain, vol. 132, pp. 213-24, Jan 2009.

2. Rodriguez, E., George, N., Lachaux, J. P., Martinerie, J., Renault, B., and Varela, F. J., "Perception's shadow: long-distance synchronization of human brain activity," Nature, vol. 397, pp. 430-3, Feb 41999.

3. Bartolomei, F., Bosma, I., Klein, M., Baayen, J. C., Reijneveld, J. C., Postma, T. J., Heimans, J. J., van Dijk, B. W., de Munck, J. C., de Jongh, A., Cover, K. S., and Stam, C. J., "How do brain tumors alter functional connectivity? A magnetoencephalography study," Ann Neurol, vol. 59, pp. 128-38, Jan 2006.

4. Brunet, D., Murray, M. M., and Michel, C. M., "Spatiotemporal analysis of multichannel EEG: CARTOOL," Comput Intell Neurosci, vol. 2011, p. 813870, 2011.

5. Murray, M. M., Brunet, D., and Michel, C. M., "Topographic ERP analyses: a step-by-step tutorial review," Brain Topogr, vol. 20, pp. 249-64, Jun 2008.

6. Koenig, T., Studer, D., Hubl, D., Melie, L., and Strik, W. K., "Brain connectivity at different time-scales measured with EEG," Philos Trans R Soc Lond B Biol Sci, vol. 360, pp. 1015-23, May 292005

7. Dimitriadis, S. I., Laskaris, N. A., Tsirka, V., Vourkas, M., Micheloyannis, S., and Fotopoulos, S., "Tracking brain dynamics via time-dependent network analysis," J Neurosci Methods, vol. 193, pp. 145-55, Oct 302010.

8. Allen, E. A., Damaraju, E., Plis, S. M., Erhardt, E. B., Eichele, T., and Calhoun, V. D., "Tracking whole-brain connectivity dynamics in the resting state," Cereb Cortex, vol. 24, pp. 663-76, Mar 2014.

9. Alario, F. X. and Ferrand, L., "A set of 400 pictures standardized for French: norms for name agreement, image agreement, familiarity, visual complexity, image variability, and age of acquisition," Behav Res Methods Instrum Comput, vol. 31, pp. 531-52, Aug 1999.

10. Delorme, A. and Makeig, S., "EEGLAB: an open source toolbox for analysis of single-trial EEG dynamics including independent component analysis," J Neurosci Methods, vol. 134, pp. 9-21, Mar 152004.

11. Lachaux, J. P., Rodriguez, E., Martinerie, J., and Varela, F. J., "Measuring phase synchrony in brain signals," Hum Brain Mapp, vol. 8, pp. 194-208, 1999.

12. Sporns, O., "Structure and function of complex brain networks," Dialogues Clin Neurosci, vol. 15, pp. 247-62, Sep 2013.

13. Salmelin, R., Hari, R., Lounasmaa, O. V., and Sams, M., "Dynamics of brain activation during picture naming," Nature, vol. 368, pp. 463-5, Mar 311994.

14. Hassan, M., Mheich, A., Wendling, F., Dufor, O., and Berrou, C., "Graph-based analysis of brain connectivity during spelling task," in 2nd IEEE International Conference on Advances in Biomedical Engineering (ICABME), Liban, Sep 2013., 2013, pp. 191-194.

Author: Ahmad Mheich

Institute: LTSI, University of Rennes 1

Street: $\quad$ Compus de Beaulieu

City: Rennes

Country: France

Mail: ahmad.mheich@univ-rennes1.fr 